\title{
Observation of Biexcitons in the presence of Trions generated via Sequential Absorption of Multiple Photons in Colloidal Quantum Dot Solids
}

\author{
Ahmet Fatih Cihan ${ }^{1}$, Pedro Ludwig Hernandez Martinez ${ }^{1,2}$, Yusuf Kelestemur ${ }^{1}$ and Hilmi Volkan Demir ${ }^{1,2}$ \\ ${ }^{1}$ Department of Electrical and Electronics Engineering, Department of Physics, UNAM - Institute of Materials Science and Nanotechnology, Bilkent \\ University, Ankara 06800 Turkey; ${ }^{2}$ School of Electrical and Electronic Engineering, School of Physical and Mathematical Sciences, Nanyang \\ Technological University, Nanyang Avenue, Singapore 639798, Singapore \\ *Tel:[+90](312) 2901021, e-mail: volkan@stanfordalumni.org
}

Multi exciton generation (MEG) and multi exciton recombination (MER) in semiconductor quantum dots (QDs) have recently attracted significant scientific interest as a possible means to improve device efficiencies [1-5]. Convenient bandgap tunability, easy colloidal synthesis, and solution-based processability of these QDs make them further attractive for such device applications using MEG and MER. For example, recent theoretical and experimental studies have shown that MEG enables $>100 \%$ peak external quantum efficiency where the generated multi excitons (MEs) are collected in a simple QD solar cell structure [1]. Furthermore, MEG has also been shown in QD photodetectors exhibiting substantially increased photocurrent levels [2]. Another promising application for MEs is the use of QDs as an alternative gain medium based on MER for lasers. Although MEG is very promising and supported with quite persuasive reports, there are still some debatable issues that need to be clarified. One of the issues that have generated great debates in the field has been the confusion of MER with the recombination of trions, which takes place in photocharged QDs. To utilize MEG and MER in practical devices such as QD solar cells and QD lasing devices, these phenomena need to be well understood. Here, we showed distinct spectrally-resolved temporal behavior of biexciton (BX), single exciton $(\mathrm{X})$ and trion radiative recombinations in near unity quantum yield (QY) quasi-type II CdSe/CdS core/shell nanocrystal QDs. Upon sequential absorption of multiple photons, the extraction of Xs, BXs, and trions were achieved using time correlated single photon counting (TCSPC) measurements performed on low concentration thin film samples of these QDs at different emission wavelengths. The QDs were embedded in PMMA medium to obtain homogeneous samples and avoid Förster-type nonradiative energy transfer (NRET) between them. Here to extract Xs, BXs, and trions, we devised a new analysis approach for the time decays of the QDs that allowed us to attribute the physical events to their corresponding time decay terms, which were further verified with their excitation intensity dependencies [6].

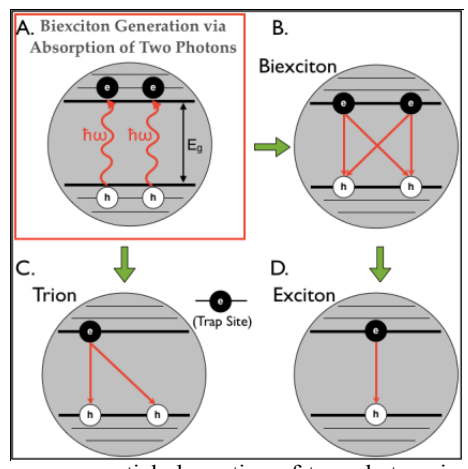

Figure 1. (A) Generation of biexcitons (BXs) upon sequential absorption of two photons in a QD with an effective bandgap of Eg. Illustration of possible recombination pathways of $(\mathbf{B})$ a BX, (C) a trion (where one of the electrons is trapped in a trap site leaving QD occupied with an electron and two holes), and (D) a single exciton (X).

The generation of MEs and their possible recombination pathways as BXs, trions and Xs are illustrated in Figure 1. Here BXs are generated by the sequential absorption of two photons in a single laser pulse. Since it has been shown that MEs generated by the absorption of two photons exhibit the same behavior as the MEs generated by the absorption of a single highly energetic photon (through a process called carrier multiplication (CM)) [3], the results of this study are also valid for the MEs generated via CM process. In this work, to suppress undesired processes that could potentially complicate the analysis, we worked at cryogenic temperatures below $20 \mathrm{~K}$. In addition to the suppression of NRET in low concentration samples and that of undesired processes at low temperatures, the QDs having near unity QY ensured that the time resolved decay components obtained from TCSPC are mostly due to radiative recombination events.

The results of time resolved fluorescence (TRF) experiments were analyzed by the least chi-square fittings of multi-exponential decays in the form of $\mathrm{A}_{1} \mathrm{e}^{-\mathrm{t} / \tau 1}+\mathrm{A}_{2} \mathrm{e}^{-\mathrm{t} / \tau 2}+\mathrm{A}_{3} \mathrm{e}^{-\mathrm{t} / \tau 3}+\mathrm{A}_{4} \mathrm{e}^{-\mathrm{t} / \tau 4}+$ background. We benefited from the significant lifetime differences of different physical events taking place in the QD ensemble to distinguish them from one another. This made it possible to study the spectral-temporal behavior of BX, trion and X recombination events by investigating the fitting terms at different emission wavelengths under different excitation intensities. To obtain the total number of happenings of each event in the excitation volume of the sample, the corresponding TRF decay terms were integrated with respect to time. This leads to $\int \mathrm{A}_{\mathrm{i}} \mathrm{e}^{-\mathrm{t} / \mathrm{i} i \mathrm{dt}}=\mathrm{A}_{\mathrm{i}} \tau_{\mathrm{i}}(i=1,2,3)$, each of which is then proportional to the total number of occurrences of the individual event represented by the weighting factor $\mathrm{A}_{i}$ and the lifetime component $\tau_{\mathrm{i}}$ in the resulting decay curve. This lifetime extraction approach has enabled us to resolve the spectral distribution of individual recombination events, which would otherwise be undistinguishable in ensemble measurements due to small spectral behavior differences. 

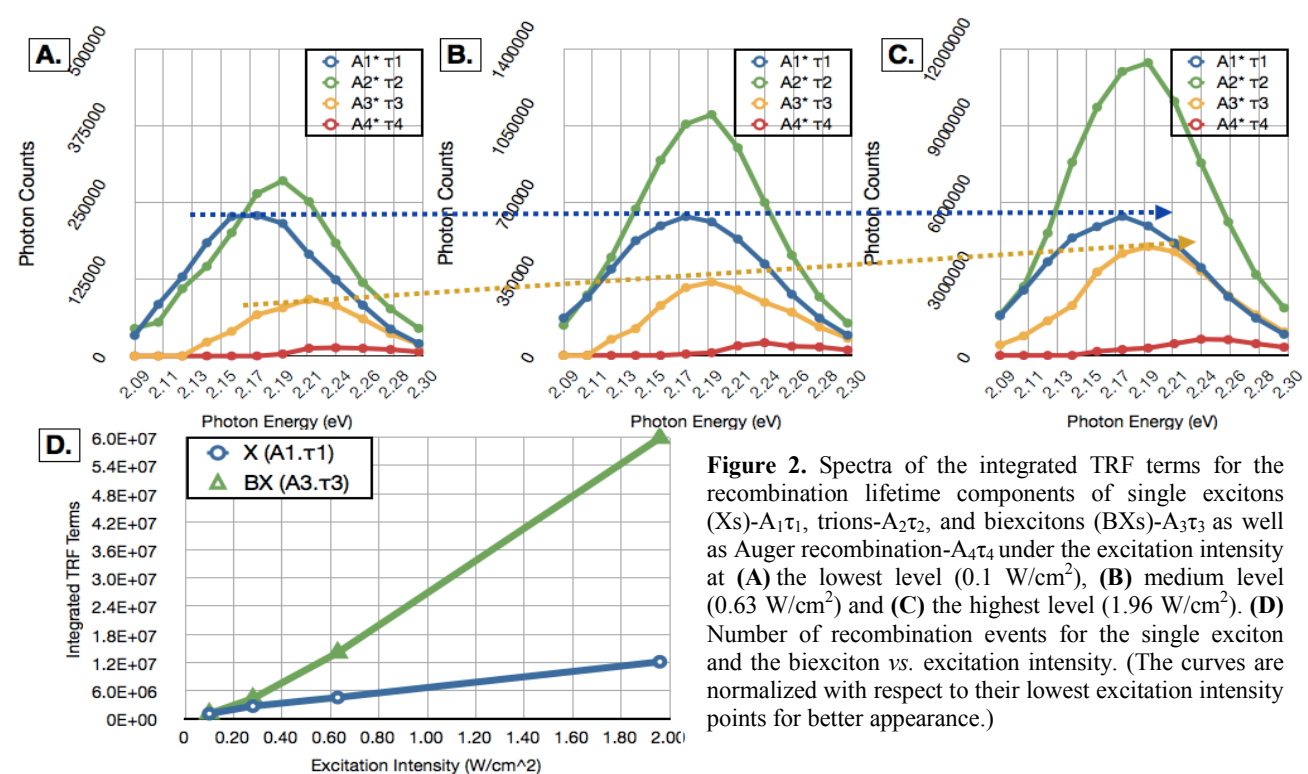

Figure 2. Spectra of the integrated TRF terms for the recombination lifetime components of single excitons $(\mathrm{Xs})-\mathrm{A}_{1} \tau_{1}$, trions- $\mathrm{A}_{2} \tau_{2}$, and biexcitons $(\mathrm{BXs})-\mathrm{A}_{3} \tau_{3}$ as well as Auger recombination- $\mathrm{A}_{4} \tau_{4}$ under the excitation intensity at (A) the lowest level $\left(0.1 \mathrm{~W} / \mathrm{cm}^{2}\right)$, (B) medium level $\left(0.63 \mathrm{~W} / \mathrm{cm}^{2}\right)$ and $(\mathbf{C})$ the highest level $\left(1.96 \mathrm{~W} / \mathrm{cm}^{2}\right)$. (D) Number of recombination events for the single exciton and the biexciton vs. excitation intensity. (The curves are normalized with respect to their lowest excitation intensity Excitation Intensity $\left(\mathrm{W} / \mathrm{cm}^{\wedge} 2\right)$

After the coefficients and lifetimes were obtained, the excitation intensity dependencies of each event were studied. The spectra of the integrated TRF decay terms are presented in Figures $2 \mathrm{~A}, 2 \mathrm{~B}$, and 2C for the lowest, medium and highest excitation intensity cases, respectively. With the spectral distributions of each integrated TRF decay term, the excitation intensity dependencies of $A_{1} \tau_{1}$ and $A_{3} \tau_{3}$ are depicted in Figure $2 \mathrm{D}$. This shows that $A_{1} \tau_{1}$ has a linear intensity dependence while $\mathrm{A}_{3} \tau_{3}$ has a quadratic intensity dependence. These dependencies are considered as strong evidences for the biexcitonic origin of the $A_{3} \tau_{3}$ term and the single excitonic origin of $A_{1} \tau_{1}$. [4] The reason why the number of BX recombination events depends quadratically on the intensity is that the probability of absorbing two photons for a QD in a single pulse quadratically increases with the intensity. It can also be inferred from the intensity dependencies of these components that, as the intensity is increased, the BXs are expected to dominate, which can also be clearly seen in the evolution of spectral distribution from Figure 2A to Figure 2C. Here the blue and yellow dashed arrows are provided to guide the eye for the contribution of the $\mathrm{BX}$ component $\mathrm{A}_{3} \tau_{3}$.

From the spectral peaks of the BX and $\mathrm{X}$ terms in Figures $2 \mathrm{~A}-2 \mathrm{C}$, we can see that the BX peak is blue-shifted with respect to the X peak, as expected for the quasi-Type II QDs. In these QDs, because of the band alignments of the core and shell materials, the X-X interaction is repulsive, which is the reason for the blue-shifted BX peak. The energy shift $\left(\Delta \mathrm{E}_{\mathrm{BX}}\right)$ between adjacent energy peaks $\mathrm{E}_{i}(i=\mathrm{X}, \mathrm{BX})$ is therefore related to the binding energies of the excitons: $\Delta \mathrm{E}_{\mathrm{BX}}=$ $2 \mathrm{E}_{\mathrm{X}}-\mathrm{E}_{\mathrm{BX}}$. Here, the energy peaks values were evaluated by the standard second-order perturbation theory and the results were found to be supporting the experimental results.

Another strong evidence for the BX lifetime component $\tau_{3}$ is its comparison to the single exciton recombination lifetime $\tau_{1}$. The lifetime analysis of the TRF decay curves automatically yielded solely based on the least chi-square fitting that the lifetime of the BX component, $\tau_{3}$, is a quarter (1/4) of the single exciton lifetime $\tau_{1}$ over the entire spectrum at all excitation intensities. This lifetime ratio is consistent with the free carrier model proposed by McGuire et al. [5] This ratio can be estimated by considering the possible recombination pathways of BXs and Xs as sketched in Figures 1B and 1D. Because of four possible recombination pathways for BXs as opposed to the single recombination possibility of Xs, the lifetime ratio of $\tau_{1} / \tau_{3}$ is expected to be 4 .

Furthermore, the least chi-square fitting gave the trion lifetime $\tau_{2}$ to be a half $(1 / 2)$ of the single exciton lifetime $\tau_{1}$ over the entire spectrum at all excitation intensities, which again agrees with the free carrier model. Besides the lifetime consistency, we also verified that $\mathrm{A}_{2} \tau_{2}$ is the trion decay term by making photocharging experiments and observed that this component becomes more pronounced for more photocharged cases. The last component of our analysis, $\mathrm{A}_{4} \tau_{4}$, is attributed mainly to the nonradiative Auger Recombination of MEs and trions because of its very short lifetime, which is about 100 s of ps.

In conclusion, in this work we obtained spectrally resolved photoluminescence time decays of BXs in our near unity efficiency quasi-type II CdSe/CdS QDs. We differentiated the BX decays from the trion decays at all emission wavelengths across the entire photoluminescence spectrum. These findings are supported by the evidences of specific decay rate ratios and characteristic intensity dependencies. We believe this clarifies the previous confusions of MEG with other processes including the decay of trions in the QDs. This work bridges the gap in the understanding of MEG for the case of $\mathrm{CdSe} / \mathrm{CdS}$ quasi-type II QDs, which offers potential applications in photovoltaic, photodetection and lasing device platforms.

Acknowledgements: This work is supported in part by EU-FP7 Nanophotonics4Energy NoE, and TUBITAK EEEAG, 109E002, 109E004, 110E010, and 110E217, and in part by NRF-CRP-6-2010-02 and NRF-RF-2009-09. H.V.D. acknowledges support from ESF-EURYI and TUBA-GEBIP; and A.F.C. from TUBITAK BIDEB.

References

[1] O. E. Semonin et al, Science, 334, 1530, 2011.

[2] V. Sukhovatkin et al., Science, 324, 1542, 2009.

[3] R. D. Schaller et al., J. Phys. Chem. B, 110, 25332, 2006.
[4] Y. Louyer, et al., Nano Letters, 11,4370, 2011.

[5] J. A. McGuire et al., Acc. Chem. Res., 41,1810, 2008.

[6] A. F. Cihan, et al. (2012) (submitted). 